\title{
Safeguarding parental identity: Dnmt1 maintains imprints during epigenetic reprogramming in early embryogenesis
}

\author{
Miguel R. Branco, Masaaki Oda, and Wolf Reik ${ }^{1}$ \\ Laboratory of Developmental Genetics and Imprinting, The Babraham Institute, Cambridge CB22 3AT, United Kingdom
}

During early mammalian embryogenesis, the genome undergoes global epigenetic reprogramming, losing most of its methylation before re-establishing it de novo at implantation. However, faithful maintenance of methylation at imprinted genes during this process is vital for embryonic development, but the DNA methyltransferase responsible for this maintenance has remained unknown. In this issue of Genes \& Development, Hirasawa and colleagues (pp. 1607-1616) show that Dnmt1, and not Dnmt3a or Dnmt3b, maintains methylation at genomic imprints during preimplantation development.

\section{Gene regulation and DNA methylation}

Genetic information alone cannot fully explain the sophisticated program of mammalian development, and dynamic regulation of gene expression is necessary to attain lineage-specific repertoires of active and silent genes at each stage of development. Epigenetic marking provides the main platform by which cells generate and maintain diverse patterns of gene expression from identical genetic information. Epigenetic marks such as DNA methylation and histone modifications influence the binding affinity of trans-acting transcription activators/repressors and recruit chromatin remodeling enzymes, thus changing the structure and function of chromatin locally. These modifications play important roles in biological processes such as $\mathrm{X}$ inactivation, genomic imprinting, and transposon silencing. As epigenetic modifications might be stably inherited through mitotic division, without changes in DNA sequence, epigenetic information can be seen as a mechanism for cellular memory.

In mammals, DNA methylation consists in the symmetric methylation of cytosine at the $5^{\prime}$ position in CpG dinucleotides and is generally associated with repressive chromatin states (Bird 2002). Three main functional mammalian DNA methyltransferases have been characterized: Dnmt1, Dnmt3a, and Dnmt3b (Chen and Li 2004).

[Keywords: Genomic imprinting; DNA methylation; pre-implantation embryos; Dnmt1]

${ }^{1}$ Corresponding author.

E-MAIL wolf.reik@bbsrc.ac.uk; FAX 441223496015.

Article is online at http://www.genesdev.org/cgi/doi/10.1101/gad.1690508.
Dnmt1 is considered to be the major enzyme responsible for maintenance of methylation during replication, given its high affinity for hemimethylated DNA in vitro. It is ubiquitously expressed in proliferating cells and found associated with replication foci during $S$ phase. The Dnmt1 gene locus encodes two isoforms of the enzyme: a somatic form, Dnmt1s, and a oocyte-specific form, Dnmtlo, which is 118 amino acids shorter at the amino terminus. Although Dnmtlo is more stable than Dnmtls, there is no functional difference between them (Ding and Chaillet 2002). Dnmt3a and Dnmt3b are both required for de novo methylation at repeat sequences, imprinted genes, and developmental genes, and are highly expressed in cells where substantial de novo methylation activity is observed; e.g., in developing germ cells and early embryonic cells (Okano et al. 1999; Kaneda et al. 2004; Oda et al. 2006).

\section{Epigenetic reprogramming of the genome}

Genome-wide analyses of DNA methylation have revealed that different tissues and cell types have distinct profiles of DNA methylation, suggesting that methylation is important for cellular identity (Bernstein et al. 2007; Farthing et al. 2008). In mammalian development, the global DNA methylation profile of the genome is dynamically reprogrammed during gametogenesis and early embryogenesis (Reik 2007). At both of these stages, the genome first undergoes global demethylation, after which de novo methylation occurs, thus re-establishing global methylation levels, but in different genomic patterns. Demethylation of the genome is generally correlated with permissive chromatin states and the acquisition of pluripotency, and conversely, methylation is correlated with repressive states and loss of pluripotency (Reik 2007).

After fertilization, the reprogramming of both maternal and paternal genomes is initiated. However, different mechanisms are involved, and while the maternal genome undergoes a stepwise, passive demethylation, the paternal genome is rapidly demethylated before the first cell division (Santos et al. 2002). Passive demethylation during early embryogenesis is presumably due to the lack of maintenance methylation activity, as Dnmt1 ap- 
pears to be excluded from the nucleus during most of preimplantation development (Howell et al. 2001). Methylation levels in the preimplantation embryo are typically at their lowest by the morula stage, and as the blastocyst is being formed they start being regained, preferentially in the inner cell mass (Dean et al. 2001; Santos et al. 2002). Importantly, however, while a large part of the genome undergoes demethylation, there are specific sequences-including the transposon family of IAPs and all imprinted genes studied so far-whose methylation is maintained (Lane et al. 2003; Edwards and FergusonSmith 2007).

\section{Control of imprinted gene expression}

Genomic imprinting plays important roles in development, especially in fetal and postnatal growth, as well as in the development and function of the placenta (Constancia et al. 2004). Many imprinted genes in the genome are clustered, and their cluster-wide allele-specific expression depends on cis-acting elements named imprinting control regions (ICRs) (Edwards and Ferguson-Smith 2007). ICRs acquire differential DNA methylation between paternal and maternal alleles, and thus DNA methylation acts as a primary mark for imprinting. During gametogenesis, imprinted methylation at ICRs is erased by an unknown but potentially active mechanism at around embryonic day 11.5 (E11.5), after primordial germ cells have arrived in the genital ridges (Hajkova et al. 2002, 2008; Lee et al. 2002), and subsequently methylation of ICRs is re-established in a sex-specific fashion. Both Dnmt3a and Dnm31, which is a member of Dnmt3 family but lacking catalytic activity, are essential for the establishment of methylation at imprinted genes in the germ cells (Bourc'his et al. 2001; Kaneda et al. 2004). In post-implantation embryos and somatic cells, maintenance of methylation at imprinted genes depends on Dnmt1 (Li et al. 1993).

During early embryogenesis, although global epigenetic reprogramming occurs, imprinted methylation is resistant to the demethylation process. It has been shown previously that the oocyte-specific Dnmtlo maintains imprints during one cell cycle alone, as mouse embryos from Dnmt1o-null females lost roughly half of their imprints (Howell et al. 2001). In accordance with this observation, Dnmtlo was found excluded from nuclei at most preimplantation stages, with the exception of the eight-cell stage, when it translocated into the nucleus for one cell cycle (Carlson et al. 1992; Howell et al. 2001; Ratnam et al. 2002). No Dnmtls was detected at least until the blastocyst stage, and therefore the question of which methyltransferase maintained genomic imprints in preimplantation stages other than the eight-cell stage remained open (Howell et al. 2001; Ratnam et al. 2002). In this issue of Genes \& Development, Hiroyuki Sasaki's group addresses and convincingly answers this question (Hirasawa et al. 2008).

\section{A job for de novo DNA methyltransferases?}

Although Dnmt3a and Dnmt3b have been mainly implicated in de novo DNA methylation during gametogen- esis and early embryogenesis, there is evidence that they also help to maintain overall methylation levels, including at imprinted genes (Okano et al. 1999; Chen et al. 2003; Jackson et al. 2004). In their study, Hirasawa et al. (2008) first explored the possibility that Dnmt3a and Dnmt3b were involved in the maintenance of methylation at genomic imprints during the epigenetic reprogramming that occurs in early embryogenesis. Previous studies suggested that while mRNAs of Dnmt3a and Dnmt3b were only detected at relatively low levels before the morula stage (Ko et al. 2005; Vassena et al. 2005), immunostaining experiments seemed to indicate that the respective proteins were present at most stages of preimplantation development (Ko et al. 2005). Hirasawa et al. (2008) have now clarified the expression of the de novo methyltransferases in early embryogenesis (summarized in Fig. 1). By using knockout embryos for Dnmt3a and $D n m t 3 b$, the investigators demonstrate the specificity of the antibodies used, thus convincingly revealing the expression pattern of the proteins. Dnmt3a expression is relatively high in the growing oocyte and fertilized embryo and then becomes progressively lower until virtually no protein is detected in the blastocyst, while conversely, Dnmt3b expression is very low in the oocyte and one- to two-cell embryo and increases throughout subsequent stages, being highly expressed in both lineages of the early blastocyst. Notably, upon expansion and implantation of the blastocyst, Dnmt3b becomes restricted to the inner cell mass (Watanabe et al. 2002). In a series of elegant experiments, Hirasawa et al. (2008) further determine where and when the de novo methyltransferase genes are first transcribed by using tissue-specific Cre recombinases that knock out the respective gene conditionally in either the male or female gametes. The investigators show that, in accordance with their expression pattern, Dnmt3a in the embryo is mainly inherited from the oocyte, while Dnmt3b is a result of embryonic transcription, which initiates at the two-cell stage.

Dnmt3a and Dnmt3b are often found to have overlapping functions and even exhibit synergism (Okano et al. 1999; Kato et al. 2007; Li et al. 2007). Thus, to test whether the presence of these enzymes in the pre-implantation embryo was needed for the maintenance of genomic imprints, Hirasawa et al. (2008) generated double knockout embryos for both de novo methyltransferases by crossing heterozygous males with homozygous females expressing the appropriate Cre recombinase. As Dnmt3a establishes maternal imprints de novo during oogenesis, knockout of the gene in the oocyte leads to a failure to establish these imprints (Kaneda et al. 2004), and therefore only paternally inherited methylation could be studied in the early double knockout embryo. The investigators found that parent-specific methylation of H19 and Dlk1/Glt2 was faithfully maintained in the absence of both Dnmt3a and Dnmt3b, showing that the de novo methyltransferases were dispensable for the maintenance of paternal imprints during epigenetic reprogramming of the embryo. Interestingly, another paternally methylated locus, Rasgrf1, lost some of its methylation 

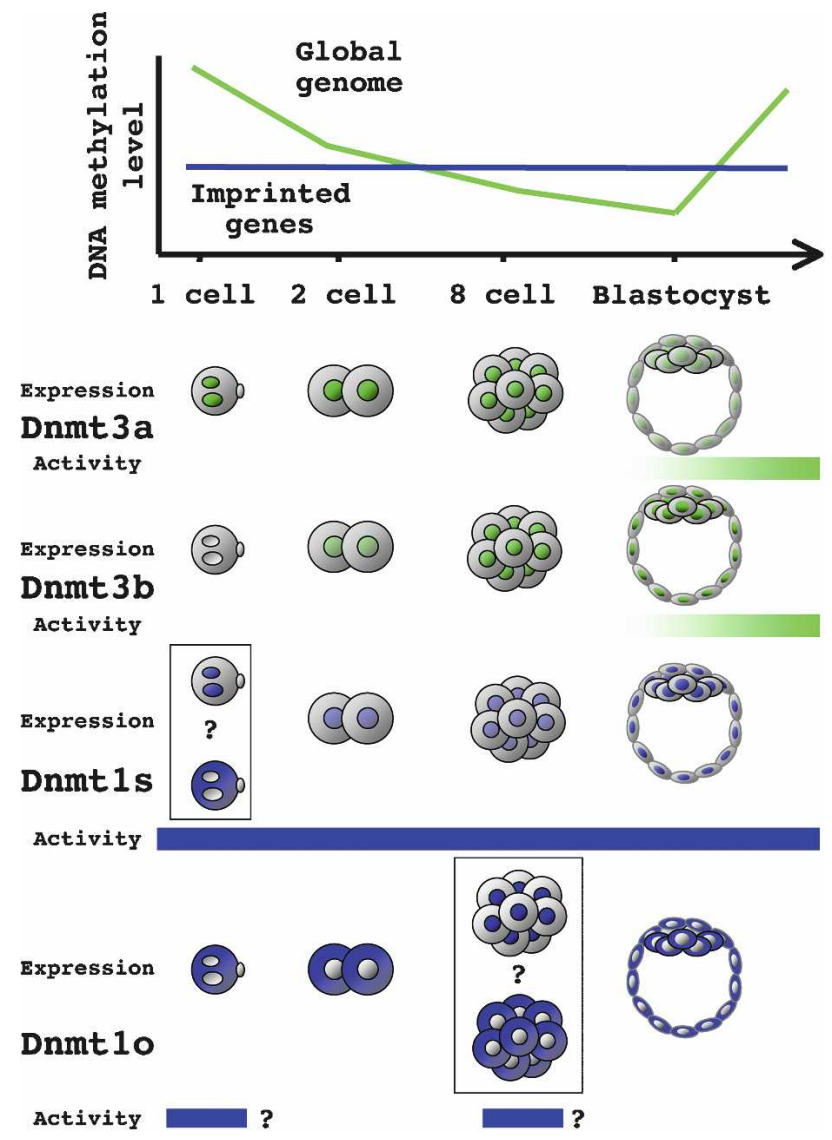

Figure 1. Expression and action of DNA methyltransferases during epigenetic reprogramming in the early mouse embryo. Throughout pre-implantation development, most of the genome's methylation (green line) is erased and later re-established in a different pattern at the blastocyst stage by the action of the de novo methyltransferases Dnmt3a and Dnmt3b. These enzymes have opposing expression patterns, with the oocytegenerated Dnmt3a becoming less expressed throughout cell divisions, and the zygote-generated Dnmt3b becoming more expressed. Activity of both enzymes is required for de novo methylation around implantation. Imprinted genes, which are vital for the development of the embryo, are resistant to demethylation (blue line). Hirasawa et al. (2008) show that the maintenance of methylation at imprints during preimplantation development is the responsibility of Dnmt1. This action is mainly carried out by the somatic form (Dnmt1s), but the oocyte form (Dnmt1o) is also necessary. However, conflicting results on the localization of Dnmtlo at the 8-cell stage make it unclear at which stage this form of the enzyme is required.

in the double knockout, suggesting that Dnmt3a and Dnmt3b may play a role in maintaining imprints at this particular locus. Hirasawa et al. (2008) suggest that the unusual repeat structure of the Rasgrf1 DMR may be the reason for the different requirement in methyltransferases.

\section{Finding Dnmt1s}

The initial suggestion that Dnmtlo was localized inside the nucleus for only one cell cycle during pre-implanta- tion development, and that Dnmt1s was undetectable, seemed to exclude the possibility that Dnmtl could be involved in the maintenance of imprinted methylation (Carlson et al. 1992; Howell et al. 2001; Ratnam et al. 2002). However, two recent studies have argued against these earlier findings, suggesting that Dnmtls could be the imprinting maintenance enzyme researchers had been looking for (Cirio et al. 2008; Kurihara et al. 2008). By using antibodies specific for the longer somatic form of Dnmt1, both studies revealed that Dnmt1s is present in nuclei of preimplantation embryos, but that it is far less abundant $(\sim 1 / 2000$ th) than the oocyte form, which is mainly localized in the cytoplasm. However, while Cirio et al. (2008) do not detect Dnmtls within pronuclei, Kurihara et al. (2008) find the maternal pool of Dnmtls associated with chromatin as early as the unfertilized egg. The cell cycle-dependent localization of the enzyme may explain these disparate results (Kurihara et al. 2008). Hirasawa et al. (2008) now confirm that Dnmtls is indeed expressed at low levels during preimplantation development and, as Kurihara et al. (2008), find no evidence for the translocation of Dnmtlo to the nucleus at the eight-cell stage. Notably, the difference in results from previous studies (Carlson et al. 1992; Howell et al. 2001) could not be attributed to a difference in the antibody used. These conflicting and puzzling observations will require further investigation. Using conditional knockout lines, Hirasawa et al. (2008) further show that Dnmtls is mainly of zygotic origin, whereas, as expected, Dnmtlo originates from the oocyte. However, a more detailed analysis by Cirio et al. (2008) has shown that a maternal pool of Dnmtls remains in the embryo until the two-cell stage, after which the embryonic pool takes over.

\section{Dnmt1s takes care of imprints}

The presence of Dnmtls in the nucleus of preimplantation embryos reopened the possibility that Dnmt1 is involved in maintaining the parent-specific methylation state of imprinted genes. In fact, Kurihara et al. (2008) had shown in their study that microinjection of antiDnmt1s antibodies or specific siRNAs into the one-cell embryo partially reduced methylation at the imprinted H19 locus. Hirasawa et al. (2008) show conclusively that Dnmtl indeed maintains DNA methylation at genomic imprints. The investigators found that knockout of Dnmt1 (both 1o and 1s forms) in embryos leads to a complete loss of methylation at both paternally and maternally methylated DMRs. Notably, Rasgrf1 also lost all of its methylation, suggesting that if Dnmt3a and Dnmt3b have a role in methylation maintenance at this locus, it is dependent on Dnmt1 activity. Conditional knockout of maternal Dnmt1 resulted in a partial loss of imprinting, similar to that observed previously for embryos from Dnmtlo-null females (Howell et al. 2001). However, as Hisawara et al. (2008) did not specifically knock out the somatic or oocyte forms of Dnmtl in their experiments, and given the presence of maternal Dnmtls until the two-cell stage (Cirio et al. 2008), it is 
possible that the maternal Dnmtls may also play a role in the maintenance of imprints. This question also becomes pertinent in light of the new evidence that Dnmtlo does not seem to translocate to the nucleus at the eight-cell stage (Hirasawa et al. 2008; Kurihara et al. 2008). Therefore, if Dnmtlo is necessary to maintain imprints for one cell cycle, then it is more likely to do so at the one-cell stage, before transcription of embryonic Dnmt1s occurs. Although Dnmtlo appears to be excluded from the nucleus at this stage, it is possible that, similar to Dnmt1s, the nuclear pool of Dnmtlo is simply less abundant than the cytoplasmic one. The question of when Dnmtlo is active could be answered by thorough stage-by-stage analyses of imprinted methylation of embryos from Dnmtlo-null females. This would be expected to reveal a $50 \%$ loss of methylation at imprints one cell division after the stage at which Dnmtlo is active. Notably, Hisawara et al. (2008) did not always observe a $50 \%$ loss of methylation in the conditional maternal Dnmt1 knockout, probably because the analyses were performed at the blastocyst stage, and clonal selection could occur due to the fact that different blastomeres inherited distinct methylation patterns.

\section{The new questions}

Apart from the question of the timing of Dnmtlo action in the preimplantation embryo, the study by Hirasawa et al. (2008) opens up exciting new questions about the role and regulation of Dnmtl during early embryogenesis. Namely, is Dnmt1s also the enzyme responsible for maintaining methylation at other sequences that are resistant to reprogramming such as IAPs (Lane et al. 2003)? Previous studies suggest that this is so and that Dnmtlo may also be involved in the process (Gaudet et al. 2004; Kurihara et al. 2008), but conclusive evidence such as the one presented here by Hirasawa et al. (2008) on genomic imprints would be desirable.

More importantly, and as Hirasawa et al. (2008) point out, the main question now is how specificity is conferred to Dnmtls's activity such that methylation is maintained at genomic imprints, but not at other sequences; namely, single-copy genes (Oswald et al. 2000) and L1 repeats (Lane et al. 2003). We have only begun to understand how Dnmt1 is targeted to hemimethylated DNA, but recent work has shown that the protein Np95 (or Uhrf1) is key to this process (Bostick et al. 2007; Sharif et al. 2007). Np95 binds to hemimethylated DNA and forms complexes with Dnmtl and PCNA, thus targeting Dnmtl activity to replication forks (Bostick et al. 2007; Sharif et al. 2007). Furthermore, it has been suggested that the fact that Np95 forms complexes with multiple other chromatin modifiers may provide a sensing mechanism for particular chromatin states, such that maintenance of methylation would be selective for these epigenetic environments (Sharif et al. 2007). Dnmt1 itself also interacts with various histone modifiers and chromatin remodelers, suggesting that its activity is likely to be dependent on previously established chromatin modifications (Burgers et al. 2002; Robertson 2002;
Esteve et al. 2006; Myant and Stancheva 2008). Nonetheless, it remains to be seen what is epigenetically unique to imprinted regions that distinguishes them from other regions that become demethylated. Another clue may come from the protein Stella, which has been shown to protect the maternal genome, and in particular imprinted genes and IAPs, from active demethylation at the one-cell stage (Nakamura et al. 2007). Although the unspecific binding of Stella to the genome suggests that other proteins are necessary for its protective role, it would be interesting to test whether Stella may also protect imprints from the passive demethylation that occurs throughout the first cell divisions of embryogenesis.

Our basic knowledge of the mechanisms involved in the methylation cycle of imprinted genes is now nearly complete, at least at the level of the enzymatic activities involved. Previous work had shown that imprints were established de novo in the germline by the action of Dnmt3a (Kaneda et al. 2004), and that after implantation of the embryo they were maintained by Dnmt1 (Li et al. 1993). Hirasawa et al. (2008) have now filled in the gap between fertilization and implantation, showing that maintenance of imprinted methylation during this phase of development is also carried out by Dnmt1. A key mystery that remains to be solved is how methylation is erased in primordial germ cells.

\section{Acknowledgments}

We thank Fátima Santos for comments on the manuscript.

\section{References}

Bernstein, B.E., Meissner, A., and Lander, E.S. 2007. The mammalian epigenome. Cell 128: 669-681.

Bird, A. 2002. DNA methylation patterns and epigenetic memory. Genes \& Dev. 16: 6-21.

Bostick, M., Kim, J.K., Esteve, P.O., Clark, A., Pradhan, S., and Jacobsen, S.E. 2007. UHRF1 plays a role in maintaining DNA methylation in mammalian cells. Science 317: 1760-1764.

Bourc'his, D., Xu, G.L., Lin, C.S., Bollman, B., and Bestor, T.H. 2001. Dnmt3L and the establishment of maternal genomic imprints. Science 294: 2536-2539.

Burgers, W.A., Fuks, F., and Kouzarides, T. 2002. DNA methyltransferases get connected to chromatin. Trends Genet. 18: $275-277$.

Carlson, L.L., Page, A.W., and Bestor, T.H. 1992. Properties and localization of DNA methyltransferase in preimplantation mouse embryos: Implications for genomic imprinting. Genes \& Dev. 6: 2536-2541.

Chen, T. and Li, E. 2004. Structure and function of eukaryotic DNA methyltransferases. Curr. Top. Dev. Biol. 60: 55-89.

Chen, T., Ueda, Y., Dodge, J.E., Wang, Z., and Li, E. 2003. Establishment and maintenance of genomic methylation patterns in mouse embryonic stem cells by Dnmt3a and Dnmt3b. Mol. Cell. Biol. 23: 5594-5605.

Cirio, M.C., Ratnam, S., Ding, F., Reinhart, B., Navara, C., and Chaillet, J.R. 2008. Preimplantation expression of the somatic form of Dnmtl suggests a role in the inheritance of genomic imprints. BMC Dev. Biol. 8: 9. doi: 10.1186/1471213X-8-9.

Constancia, M., Kelsey, G., and Reik, W. 2004. Resourceful im- 
printing. Nature 432: 53-57.

Dean, W., Santos, F., Stojkovic, M., Zakhartchenko, V., Walter, J., Wolf, E., and Reik, W. 2001. Conservation of methylation reprogramming in mammalian development: Aberrant reprogramming in cloned embryos. Proc. Natl. Acad. Sci. 98: 13734-13738.

Ding, F. and Chaillet, J.R. 2002. In vivo stabilization of the Dnmt1 (cytosine-5)-methyltransferase protein. Proc. Natl. Acad. Sci. 99: 14861-14866.

Edwards, C.A. and Ferguson-Smith, A.C. 2007. Mechanisms regulating imprinted genes in clusters. Curr. Opin. Cell Biol. 19: $281-289$.

Esteve, P.O., Chin, H.G., Smallwood, A., Feehery, G.R., Gangisetty, O., Karpf, A.R., Carey, M.F., and Pradhan, S. 2006. Direct interaction between DNMT1 and G9a coordinates DNA and histone methylation during replication. Genes \& Dev. 20: 3089-3103.

Farthing, C.R., Ficz, G., Ng, R.K., Chan, C.F., Andrews, S., Dean, W., Hemberger, M., and Reik, W. 2008. Global mapping of DNA methylation in mouse promoters reveals epigenetic reprogramming of pluripotency genes. PLoS Genet. (in press).

Gaudet, F., Rideout III, W.M., Meissner, A., Dausman, J., Leonhardt, H., and Jaenisch, R. 2004. Dnmt1 expression in preand postimplantation embryogenesis and the maintenance of IAP silencing. Mol. Cell. Biol. 24: 1640-1648.

Hajkova, P., Erhardt, S., Lane, N., Haaf, T., El-Maarri, O., Reik, W., Walter, J., and Surani, M.A. 2002. Epigenetic reprogramming in mouse primordial germ cells. Mech. Dev. 117: 1523.

Hajkova, P., Ancelin, K., Waldmann, T., Lacoste, N., Lange, U.C., Cesari, F., Lee, C., Almouzni, G., Schneider, R., and Surani, M.A. 2008. Chromatin dynamics during epigenetic reprogramming in the mouse germ line. Nature 452: 877881.

Hirasawa, R., Chiba, H., Kaneda, M., Tajima, S., Li, E., Jaenisch, R., and Sasaki, H. 2008. Maternal and zygotic Dnmtl are necessary and sufficient for the maintenance of DNA methylation imprints during preimplantation development. Genes \& Dev. (this issue), doi: 10.1101/gad.1667008.

Howell, C.Y., Bestor, T.H., Ding, F., Latham, K.E., Mertineit, C., Trasler, J.M., and Chaillet, J.R. 2001. Genomic imprinting disrupted by a maternal effect mutation in the Dnmt1 gene. Cell 104: 829-838.

Jackson, M., Krassowska, A., Gilbert, N., Chevassut, T., Forrester, L., Ansell, J., and Ramsahoye, B. 2004. Severe global DNA hypomethylation blocks differentiation and induces histone hyperacetylation in embryonic stem cells. Mol. Cell. Biol. 24: 8862-8871.

Kaneda, M., Okano, M., Hata, K., Sado, T., Tsujimoto, N., Li, E., and Sasaki, H. 2004. Essential role for de novo DNA methyltransferase Dnmt3a in paternal and maternal imprinting. Nature 429: 900-903.

Kato, Y., Kaneda, M., Hata, K., Kumaki, K., Hisano, M., Kohara, Y., Okano, M., Li, E., Nozaki, M., and Sasaki, H. 2007. Role of the Dnmt3 family in de novo methylation of imprinted and repetitive sequences during male germ cell development in the mouse. Hum. Mol. Genet. 16: 2272-2280.

Ko, Y.G., Nishino, K., Hattori, N., Arai, Y., Tanaka, S., and Shiota, K. 2005. Stage-by-stage change in DNA methylation status of Dnmt1 locus during mouse early development. J. Biol. Chem. 280: 9627-9634.

Kurihara, Y., Kawamura, Y., Uchijima, Y., Amamo, T., Kobayashi, H., Asano, T., and Kurihara, H. 2008. Maintenance of genomic methylation patterns during preimplantation development requires the somatic form of DNA methyltrans- ferase 1. Dev. Biol. 313: 335-346.

Lane, N., Dean, W., Erhardt, S., Hajkova, P., Surani, A., Walter, J., and Reik, W. 2003. Resistance of IAPs to methylation reprogramming may provide a mechanism for epigenetic inheritance in the mouse. Genesis 35: 88-93.

Lee, J., Inoue, K., Ono, R., Ogonuki, N., Kohda, T., KanekoIshino, T., Ogura, A., and Ishino, F. 2002. Erasing genomic imprinting memory in mouse clone embryos produced from day 11.5 primordial germ cells. Development 129: 1807-1817.

Li, E., Beard, C., and Jaenisch, R. 1993. Role for DNA methylation in genomic imprinting. Nature 366: 362-365.

Li, J.Y., Pu, M.T., Hirasawa, R., Li, B.Z., Huang, Y.N., Zeng, R., Jing, N.H., Chen, T., Li, E., Sasaki, H., et al. 2007. Synergistic function of DNA methyltransferases Dnmt3a and Dnmt3b in the methylation of Oct4 and Nanog. Mol. Cell. Biol. 27: 8748-8759.

Myant, K. and Stancheva, I. 2008. LSH cooperates with DNA methyltransferases to repress transcription. Mol. Cell. Biol. 28: 215-226.

Nakamura, T., Arai, Y., Umehara, H., Masuhara, M., Kimura, T., Taniguchi, H., Sekimoto, T., Ikawa, M., Yoneda, Y., Okabe, M., et al. 2007. PGC7/Stella protects against DNA demethylation in early embryogenesis. Nat. Cell Biol. 9: 64-71.

Oda, M., Yamagiwa, A., Yamamoto, S., Nakayama, T., Tsumura, A., Sasaki, H., Nakao, K., Li, E., and Okano, M. 2006. DNA methylation regulates long-range gene silencing of an X-linked homeobox gene cluster in a lineage-specific manner. Genes \& Dev. 20: 3382-3394.

Okano, M., Bell, D.W., Haber, D.A., and Li, E. 1999. DNA methyltransferases Dnmt3a and Dnmt3b are essential for de novo methylation and mammalian development. Cell 99: 247257.

Oswald, J., Engemann, S., Lane, N., Mayer, W., Olek, A., Fundele, R., Dean, W., Reik, W., and Walter, J. 2000. Active demethylation of the paternal genome in the mouse zygote. Curr. Biol. 10: 475-478.

Ratnam, S., Mertineit, C., Ding, F., Howell, C.Y., Clarke, H.J., Bestor, T.H., Chaillet, J.R., and Trasler, J.M. 2002. Dynamics of Dnmt1 methyltransferase expression and intracellular localization during oogenesis and preimplantation development. Dev. Biol. 245: 304-314.

Reik, W. 2007. Stability and flexibility of epigenetic gene regulation in mammalian development. Nature 447: 425-432.

Robertson, K.D. 2002. DNA methylation and chromatin-Unraveling the tangled web. Oncogene 21: 5361-5379.

Santos, F., Hendrich, B., Reik, W., and Dean, W. 2002. Dynamic reprogramming of DNA methylation in the early mouse embryo. Dev. Biol. 241: 172-182.

Sharif, J., Muto, M., Takebayashi, S., Suetake, I., Iwamatsu, A., Endo, T.A., Shinga, J., Mizutani-Koseki, Y., Toyoda, T., Okamura, K., et al. 2007. The SRA protein Np95 mediates epigenetic inheritance by recruiting Dnmt1 to methylated DNA. Nature 450: 908-912.

Vassena, R., Dee Schramm, R., and Latham, K.E. 2005. Speciesdependent expression patterns of DNA methyltransferase genes in mammalian oocytes and preimplantation embryos. Mol. Reprod. Dev. 72: 430-436.

Watanabe, D., Suetake, I., Tada, T., and Tajima, S. 2002. Stageand cell-specific expression of Dnmt3a and Dnmt3b during embryogenesis. Mech. Dev. 118: 187-190. 


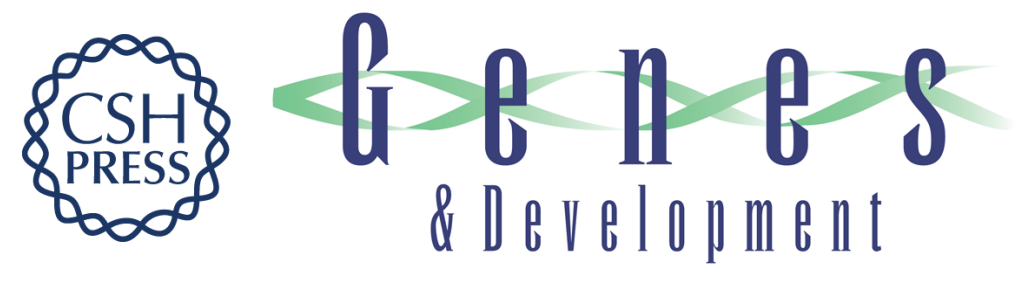

\section{Safeguarding parental identity: Dnmt1 maintains imprints during epigenetic reprogramming in early embryogenesis}

Miguel R. Branco, Masaaki Oda and Wolf Reik

Genes Dev. 2008, 22:

Access the most recent version at doi:10.1101/gad.1690508

Related Content

Maternal and zygotic Dnmt1 are necessary and sufficient for the maintenance of DNA methylation imprints during preimplantation development Ryutaro Hirasawa, Hatsune Chiba, Masahiro Kaneda, et al. Genes Dev. June , 2008 22: 1607-1616

References This article cites 40 articles, 15 of which can be accessed free at: http://genesdev.cshlp.org/content/22/12/1567.full.html\#ref-list-1

Articles cited in:

http://genesdev.cshlp.org/content/22/12/1567.full.html\#related-urls

\section{License}

Email Alerting

Service

Receive free email alerts when new articles cite this article - sign up in the box at the top right corner of the article or click here.

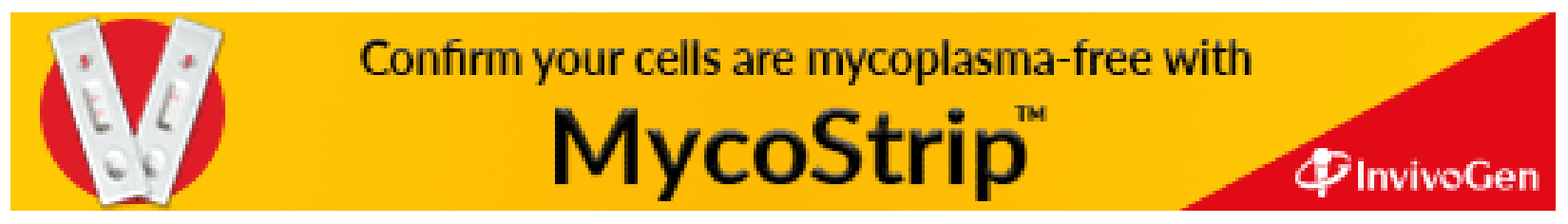

